\title{
Clinical characteristics and antimicrobial susceptibility of Bacillus cereus blood stream infections
}

\author{
Mahoko Ikeda' ${ }^{1}$ Yuka Yagihara' ${ }^{1}$ Keita Tatsuno ${ }^{1}$, Mitsuhiro Okazaki², Shu Okugawa ${ }^{1 *}$ and Kyoji Moriya
}

\begin{abstract}
Background: Bacillus cereus is one of the pathogens causing nosocomial bloodstream infections (BSIs). However, few reports have documented the antimicrobial susceptibility and clinical characteristics of Bacillus cereus BSI and the importance of empirical therapy. The aim of this study was to investigate the clinical characteristics and antimicrobial susceptibility of $B$. cereus isolates from patients with BSI and to analyze the impact of appropriate empirical therapy on the outcome of patients with B. cereus BSI.
\end{abstract}

Methods: All adult cases of bacteremia between April 2003 and March 2012 in a teaching hospital in Tokyo, Japan were reviewed retrospectively. Clinical data were collected from the patients' medical records and charts. Antimicrobial susceptibility testing was performed by broth microdilution method. The patients with B. cereus BSI were divided into an appropriate empirical therapy group and an inappropriate empirical therapy group. The primary outcome was all-cause mortality at 4 weeks after the start of BSI. The secondary outcome was early defervescence within 2 days after starting empirical therapy.

Results: There were 29 B. cereus bloodstream infection cases. No vancomycin, gentamicin, and imipenem-resistant isolates were found. However, $65.5 \%$ were resistant to clindamycin and $10.3 \%$ were resistant to levofloxacin. The main etiology was venous catheter-related (69\%). All-cause mortality at 4 weeks was not significantly different between the appropriate empirical therapy group (9 cases) and the inappropriate group (20 cases) in this study. However, early defervescence within 2 days after starting empirical therapy was significantly different $(p=0.032)$.

Conclusions: The BSI of B. cereus is mostly caused by venous catheter-related infections. Appropriate empirical therapy is important to achieve early clinical resolution in B. cereus BSI. Vancomycin is one of the appropriate selections of empirical therapy for B. cereus BSI.

Keywords: Bacillus cereus, Blood stream infection, Empirical therapy, Susceptibility

\section{Background}

The Bacillus cereus group contains six species, B. cereus, B. thuringiensis, B. weihenstephanensis, B. mycoides, $B$. pseudomycoides, and $B$. anthracis [1]. The most prevalent human pathogen in the B. cereus group is B. cereus [1]. The automated identification system in diagnostic laboratories cannot distinguish between $B$. cereus and $B$. thuringiensis, because they are genetically similar species.

\footnotetext{
*Correspondence: okugawa-tky@umin.ac.jp

${ }^{1}$ Department of Infection Control and Prevention, Faculty of Medicine,

The University of Tokyo, 7-3-1 Hongo, Bunkyo, Tokyo 113-8655, Japan

Full list of author information is available at the end of the article
}

However, B. thuringiensis is an extremely rare human pathogen and is basically an insect pathogen [2].

$B$. cereus is a spore-forming Gram-positive bacillus that exists ubiquitously in soil, marine environments, vegetables, the intestinal tracts of invertebrates, and human skin [3]. The organism is a common pathogen in food poisoning [3]. Invasive B. cereus infections such as bacteremia, pneumonia [4], eye infection [5], central nervous system (CNS) infections [6,7], and soft tissue infections $[8,9]$ have been reported in hospital settings. More information about the clinical characteristics of 
B. cereus bloodstream infection (BSI) is urgently needed to improve the treatment of patients with $B$. cereus infection.

BSIs are important causes of morbidity and mortality. Appropriate empirical therapy should be started immediately after BSI is identified [10]. Recent reports have analyzed risk factors for $B$. cereus BSI, such as prior antimicrobial treatment $[11,12]$, reuse of contaminated towels [13, 14], central venous catheter insertions [12, 15], and hematological malignancy [16]. Fatal outcomes have been related to neutropenia [6], delays in treatment [17], and CNS symptoms $[15,17]$.

Inappropriate antibiotic therapy has been shown to be predictive of higher mortality rates in patients with bacteremia compared to appropriate therapy [10]. The antimicrobial agents for the empirical therapy should be selected according to the antimicrobial susceptibility of the pathogen. However, few studies have reported on the clinical characteristics of B. cereus BSI and the importance of empirical therapy $[17,18]$. The aim of this study was therefore to investigate the antimicrobial susceptibility of clinical B. cereus isolates from patients with BSI and to analyze the impact of appropriate empirical therapy on clinical outcomes in patients with B. cereus BSI.

\section{Methods}

\section{Study setting and patients}

All cases of bacteremia that occurred between April 2003 and March 2012 in the microbial blood culture database of the University of Tokyo Hospital, a 1217-bed tertiarycare teaching hospital in Tokyo, Japan, were retrospectively reviewed. Patients with $B$. cereus bacteremia were divided into appropriate empirical therapy and inappropriate empirical therapy groups.

\section{Data collection and definitions}

All adult (age $\geq 18$ years) patients from whose blood cultures $B$. cereus was isolated were enrolled. Bacteremia caused by $B$. cereus was defined as: (1) two or more positive blood cultures collected in one day; or (2) positive blood cultures collected on more than two consecutive days. Untreated cases and cases that received oral antimicrobial therapy were excluded, as were patients who were afebrile despite positive blood cultures for B. cereus.

Patients' data, clinical symptoms, laboratory results, antibiotics used, in-hospital mortality due to any cause, presence of blood cultures that became negative, and microbiological data were collected from the clinical charts and the laboratory database. Patients' data included age, sex, underlying disease (diabetes mellitus, cirrhosis, malignancy, immunosuppressants, and neutropenia), and implanted medical devices such as central venous catheters, biliary stents, and artificial joints.
Clinical symptoms included fever, suspected focus of infection, and complications with BSI. Laboratory data were white blood cell counts, neutrophil counts, C-reactive protein and albumin levels. Data of antibiotics used before and after blood culture samples were obtained were also reviewed.

Fever was defined as a body temperature $\geq 37.5{ }^{\circ} \mathrm{C}$. When a body temperature of $<37.5{ }^{\circ} \mathrm{C}$ was maintained for $>24 \mathrm{~h}$, the patient was defined as afebrile. Body temperature was measured at least 3 times a day (at 08:00, 14:00, and 20:00). A complication was considered a focus of infection accompanied by $B$. cereus BSI.

Empirical therapy was defined as the antibiotics received on the first day of therapy for the BSI. Appropriate antimicrobial therapy was defined as systemic administration of at least one antimicrobial agent to which the $B$. cereus isolate proved susceptible in vitro.

\section{Microbiological methods}

Blood culture specimens were inoculated into BACTEC standard culture bottles in a BACTEC 9000 system (Becton, Dickinson and Company, Franklin Lakes, NJ, USA). All isolates were identified as $B$. cereus/B. thuringiensis using a VITEK2 system with the BCL card (SYSMEX bioMerieux, Tokyo, Japan). Antimicrobial susceptibility was determined with the Walkaway system and the standard criteria of the Clinical and Laboratory Standards Institute (CLSI) guide. The breakpoints against $B$. cereus in the CLSI guideline M45A2E [19] were used for the following agents: ampicillin, cefazolin, cefotaxime, caftazidime, imipenem, vancomycin, amikacin, gentamicin, erythromycin, levofloxacin, clindamycin, chloramphenicol and rifampin. For other antimicrobials, the breakpoints for Staphylococcus spp. in the CLSI guideline M 100-S22 [20] and S24 [21] were used [22].

\section{Clinical outcomes}

The primary outcome was all-cause mortality at 4 weeks after onset of bacteremia. The secondary outcome was early defervescence within 2 days after starting empirical therapy.

\section{Statistical analysis}

Fisher's exact test was used for analysis of categorical data. Non-parametric data were analyzed using the Mann-Whitney U test. Values of $P<0.05$ were considered significant. All statistical analyses were performed using JMP Pro version 10 software (SAS Institute, Cary, $\mathrm{NC}, \mathrm{USA})$.

\section{Results}

Patients' characteristics

A total of 5894 cases with positive blood cultures was identified over the 9-year study period. Of these, $B$. 
cereus was isolated in 203 cases. One hundred seventy one cases were excluded, because they had one $B$. cereus-positive blood culture. Three cases with more than two B. cereus-positive blood cultures were also excluded, because one case was untreated, one case was treated with oral antibiotics, and one case was afebrile. After applying the exclusion criteria, 29 cases $(14.3 \%$ of cases in which B. cereus was detected, $0.49 \%$ of all culture-positive cases) were included in this study. The patients' clinical characteristics are shown in Table 1. The mean age was 65.3 years (range 18-89 years), and $55.2 \%$ were male. The main etiology was venous catheter-related (69\%). The appropriate and inappropriate empirical therapy groups had similar baseline characteristics, sources of infection, and comorbidities that could significantly affect the clinical outcome. Complications except focuses of infection cited in Table 1 was endophthalmitis (1 case) occurred in the inappropriate empirical therapy group. In laboratory results, patients in the appropriate therapy group had a tendency to have higher white blood cell counts than patients in the inappropriate therapy group.

\section{Antimicrobial susceptibility}

All isolates showed sensitivity to vancomycin, gentamicin, and imipenem. However, $48.3-100 \%$ of isolates were resistant to cephalosporins, $65.5 \%$ were resistant to clindamycin, and $10.3 \%$ were resistant to levofloxacin (Table 2).

\section{Antibiotic treatment}

In the appropriate empirical therapy group, piperacillintazobactam (3 cases), vancomycin (2 cases), or ampicillin-sulbactam, cefmetazole, clindamycin, amikacin, or cefoperazone-sulbactam (1 case each) was used. In the inappropriate empirical therapy group, cefepim $(6$ cases), ampicillin-sulbactam, cefazolin, ceftriaxone/cefotaxim (3 cases each), ceftazidime ( 2 cases), cefotiam, or clindamycin (1 case each) was used, and in vitro testing showed that the $B$. cereus isolated on blood culture was resistant. One patient was not treated empirically with antibiotics. The duration of antimicrobial therapy did not differ between the two groups (Table 3). All patients, but one, were changed to an appropriate antibiotic agent by 5 days.

Table 1 Characteristics of patients with Bacillus cereus bloodstream infection

\begin{tabular}{|c|c|c|c|c|}
\hline \multirow[t]{2}{*}{ Variable } & \multirow[t]{2}{*}{ All patients $(n=29)$} & \multicolumn{2}{|c|}{ Empirical therapy that was deemed } & \multirow[t]{2}{*}{$P$ value } \\
\hline & & Appropriate $(n=9)$ & Inappropriate $(n=20)$ & \\
\hline Age, years (median, range) & $65.3(18-89)$ & $68(18-89)$ & $65(47-83)$ & 0.743 \\
\hline Sex (male/female) & $16 / 13$ & $4 / 5$ & $12 / 8$ & 0.688 \\
\hline \multicolumn{5}{|l|}{ No. of patients with comorbidity } \\
\hline Diabetes mellitus & 8 & 3 & 5 & 0.675 \\
\hline Malignancy & 15 & 4 & 11 & 0.700 \\
\hline Liver cirrhosis & 4 & 1 & 3 & 1.00 \\
\hline Immunosuppressant & 5 & 2 & 3 & 0.633 \\
\hline Neutropenia & 4 & 0 & 4 & 0.280 \\
\hline \multicolumn{5}{|c|}{ No. of patients with implanted device } \\
\hline Central venous catheter & 6 & 1 & 5 & 0.633 \\
\hline Peripheral venous catheter & 22 & 8 & 14 & 0.382 \\
\hline Others & 7 & 3 & 4 & 0.642 \\
\hline \multicolumn{5}{|l|}{ Source of BSI } \\
\hline Total catheter-related infection & 20 & 7 & 13 & 0.675 \\
\hline Peripheral blood catheter & 15 & 6 & 9 & 0.427 \\
\hline Central venous catheter & 5 & 1 & 4 & 1.00 \\
\hline Others $^{\mathrm{a}}$ & 5 & 0 & 5 & 0.153 \\
\hline Unknown & 4 & 2 & 2 & 0.568 \\
\hline \multicolumn{5}{|l|}{ Laboratory data (average, range) } \\
\hline White blood cell count $(/ \mu \mathrm{L})$ & $7497(100-22,200)$ & $9630(1800-22,200)$ & $6537(100-16,100)$ & 0.052 \\
\hline C-reactive protein $(\mathrm{g} / \mathrm{dL})$ & $4.19(0.04-16.6)$ & $3.79(0.04-16.6)$ & $3.34(0.17-13.7)$ & 0.514 \\
\hline Albumin (g/dL) & $3.29(2.10-4.20)$ & $2.71(2.10-3.90)$ & $3.45(2.40-4.20)$ & 0.206 \\
\hline
\end{tabular}

a Other sources of BSI in the inappropriate empirical therapy group include febrile neutropenia (2 cases), possible infective endocarditis according to modified Duke's criteria ( 2 cases), and peritonitis ( 1 case) 
Table 2 Antimicrobial susceptibility of major antibiotics against Bacillus cereus

\begin{tabular}{|c|c|c|c|c|c|c|c|}
\hline \multirow[t]{2}{*}{ Antimicrobial agent } & \multirow[t]{2}{*}{$\mathbf{n}$} & \multicolumn{3}{|l|}{$\mathrm{MIC}^{\mathrm{a}}(\mathrm{mg} / \mathrm{dL})$} & \multicolumn{3}{|c|}{ Interpretation n (\%) } \\
\hline & & Range & $50 \%^{b}$ & $90 \%^{b}$ & Susceptible & Interpretive & Resistant \\
\hline Vancomycin & 29 & $\leq 2$ & $\leq 2$ & $\leq 2$ & $29(100)$ & 0 & 0 \\
\hline Imipenem & 29 & $\leq 0.5$ to 4 & $\leq 0.5$ & $\leq 0.5$ & $29(100)$ & 0 & 0 \\
\hline Gentamicin & 29 & $\leq 2$ to 4 & $\leq 2$ & 4 & $29(100)$ & 0 & 0 \\
\hline Amikacin & 26 & $\leq 16$ & $\leq 16$ & $\leq 16$ & $26(100)$ & 0 & 0 \\
\hline Linezolid & 25 & $\leq 0.25$ to 2 & 1 & 1 & $25(100)$ & & \\
\hline Chloramphenicol & 22 & 2 to 4 & 4 & 4 & $22(100)$ & 0 & 0 \\
\hline Rifampin & 15 & $\leq 1$ & $\leq 1$ & $\leq 1$ & $15(100)$ & 0 & 0 \\
\hline Levofloxacin & 29 & $\leq 0.12$ to $>16$ & $\leq 0.5$ & $\leq 0.5$ & $26(89.7)$ & $1(3.4)$ & $2(6.9)$ \\
\hline Clindamycin & 29 & $<0.25$ to $\geq 4$ & 2 & 2 & $10(34.5)$ & $18(62.1)$ & $1(3.4)$ \\
\hline Erythromycin & 29 & $\leq 0.25$ to $>16$ & 0.5 & 1 & $18(62.1)$ & $6(20.7)$ & $2(6.9)$ \\
\hline Cefazolin & 29 & $\leq 8$ to $\geq 64$ & $\leq 8$ & & $15(51.7)$ & $6(20.7)$ & $8(27.6)$ \\
\hline Daptomycin & 22 & $\leq 0.12$ to 4 & 2 & 4 & $8(36.4)$ & $12(54.5)^{c}$ & \\
\hline Cefotaxime & 22 & 4 to $\geq 64$ & 16 & 32 & $2(9.1)$ & $18(81.8)$ & $2(9.1)$ \\
\hline Ampicillin/sulbactam & 25 & $\leq 0.25$ to $>8$ & 4 & $>8$ & $1(4)$ & - & $24(96)$ \\
\hline Ampicillin & 29 & 0.5 to $>8$ & $>4$ & $>4$ & 0 & - & $29(100)$ \\
\hline Ceftazidime & 22 & 32 to $>64$ & $>64$ & $>64$ & 0 & 0 & $22(100)$ \\
\hline
\end{tabular}

a Minimum inhibitory concentration

b MIC at which 50 or $90 \%$ of tested isolates are inhibited

" This isolates were interpreted as "not susceptible" because the breakpoint was only set for susceptible

Table 3 Antibiotic empirical therapy and outcomes of Bacillus cereus bloodstream infection

\begin{tabular}{|c|c|c|c|c|}
\hline \multirow[t]{2}{*}{ Variable } & \multirow[t]{2}{*}{ All patients $(n=29)$} & \multicolumn{2}{|c|}{ Empirical therapy that was deemed } & \multirow[t]{2}{*}{$P$ value } \\
\hline & & Appropriate $(n=9)$ & Inappropriate $(n=20)$ & \\
\hline $\begin{array}{c}\text { Duration of appropriate antibiotic } \\
\text { treatment median (days, range) }\end{array}$ & $15(7-41)$ & $13(7-20)$ & $16.5(7-41)$ & 0.050 \\
\hline Early defervescence (patients) & 10 & 6 & 4 & $0.032^{*}$ \\
\hline Survival at 4 weeks (patients) & $27^{\mathrm{a}}$ & $8 / 9(88.9 \%)$ & $19 / 19(100 \%)$ & 0.321 \\
\hline
\end{tabular}

* Statistically significant $(p<0.05)$

a One patient was excluded in this analysis because of transfer to another hospital

\section{Clinical outcome}

In terms of the primary outcome, no significant difference in all-cause mortality at 4 weeks after onset of bacteremia was seen between the groups (Table 3). However, the secondary outcome of early defervescence was achieved significantly more often in the appropriate empirical therapy group. Central venous catheters were all removed in both groups. The presence of blood cultures that became negative was confirmed in 6 patients of the appropriate therapy group and 17 patients of the inappropriate therapy group. However, the intervals of taking blood cultures differed depending on the cases.

\section{Discussion}

The present data show that the BSI of B. cereus was mostly caused by venous catheter-related infections and that appropriate empirical therapy is important to achieve early clinical resolution in $B$. cereus bloodstream infection, although the appropriateness of therapy does not affect mortality.

In this study, cases detected B. cereus in more than two sets of blood cultures were enrolled as definite BSI and accounted for $14.3 \%$ of B. cereus-detected cases. In our hospital, the rate of obtaining two sets of blood cultures was around $60 \%$ at the beginning of the study period, and it increased to $75 \%$ at the end. Although a further 
increase of the two-set rate may enable us to obtain more cases and uncover the detailed clinical picture of $B$. cereus $\mathrm{BSI}$, the criterion used to select the definitive $B$. cereus BSI cases was more than two sets of positive blood cultures according to the BSI guideline [23]. B. cereus bacteremia was reported to be underestimated as contamination [24, 25], and it has recently been increasingly recognized that some cases of $B$. cereus bacteremia are definite BSIs $[15,17]$.

Bacteremia due to other bacteria, such as Staphylococcus aureus and extended-spectrum beta lactamase-producing Gram-negative bacteria, caused higher 30-day mortality in patients with various comorbidities than $B$. cereus bacteremia even when empirical therapy was inappropriate [26, 27]. In the present study, only one patient died in the appropriate therapy group, and no patient died in the inappropriate therapy group, indicating that $B$. cereus might be less virulent to humans. However, the appropriateness of therapy still has benefit for patients with $B$. cereus BSI, because early defervescence was significantly related to appropriate empirical antibiotic therapy.

Which antibiotic agents are appropriate for bacteremia due to $B$. cereus? In the present study, the $B$. cereus isolated was frequently not susceptible to empirical therapy, but an early treatment response was related to susceptible empirical therapy.

In this study, all isolates were resistant to ampicillin, and almost all were resistant to cephalosporins. All were sensitive to carbapenems. This could be explained by the fact that $B$. cereus is genetically resistant to all betalactams except carbapenems [3]. However, some reports showed carbapenem-resistant B. cereus bacteremia [28, 29 ], and some in vitro studies showed that $B$. cereus possesses metallo-beta lactamase genetically [30, 31]. Thus, further study is needed to determine whether carbapenems could be one of the choices for empirical treatment of $B$. cereus BSI.

Fluoroquinolones and clindamycin are also possible if a $B$. cereus isolate is susceptible. The present data showed a resistance rate of $65.5 \%$ (19/29 cases) for clindamycin and $10.3 \%$ (3/29) for levofloxacin. Horii et al. reported that $26 \mathrm{~B}$. cereus isolates from blood cultures showed MIC90 of levofloxacin as 4 (CLSI's breakpoint of levofloxacin was $\leq 2$ as sensitive) [11]. When empirical therapy is started with these agents, the susceptibility pattern should be considered.

All isolates showed good susceptibility to vancomycin according to CLSI's breakpoint (MIC $\leq 4$ as sensitive). Other reports [11, 22] also showed no vancomycinresistant $B$. cereus isolated from both clinical samples and environmental samples. Given that a main cause of $B$. cereus BSI is catheter-related infection, vancomycin is suitable for empirical treatment of B. cereus BSI, because the common causative pathogens of catheterrelated infection are Gram-positive bacteria including $S$. aureus and coagulase-negative Staphylococci. However, BSI caused by $B$. cereus with vancomycin-reduced susceptibility (MIC $4 \mu \mathrm{g} / \mathrm{mL}$ ) was reported in neonates [32]. We should pay attention to changes in susceptibility to vancomycin.

Other optimal choices for Gram-positive bacteria, daptomycin and linezolid, are options in B. cereus BSI. In the present study, the MIC90 of linezolid was 1, and all isolates were considered susceptible. In contrast, more than half of the B. cereus showed the MIC of daptomycin $>1$, although breakpoint of daptomycin was $\leq 1$ as susceptible. The MIC of daptomycin was tested by microdilution assay. Luna et al. [22] reported that the MICs of daptomycin were $0.25-1$ using the $E$ test for 42 strains of $B$. cereus collected from the environment. The differences in the MICs might be due to differences in methodology or differences in whether they were clinical isolates or not. An in vitro study reported that a gene that gave daptomycin resistance was activated under swarming condition [33], and that spores were resistant to daptomycin until they germinated [34]. Thus, further study of the measurement of daptomycin MIC and the use of daptomycin for treatment is needed.

This study has some limitations. First, whether antimicrobials were susceptible was determined by antimicrobial breakpoints of $B$. cereus in CLSI's guideline M45A2E [19]. However, for some antimicrobials for which breakpoints have not been determined, breakpoints of S. aureus in M100 [21] were used with reference to a previous report [22]. Although breakpoints of these species were similar for various classes of antimicrobials such as cephalosporins, vancomycin was slightly different. This may be limitation of this study because undefined breakpoints of $B$. cereus may differ from those of $S$. aureus already defined in M100 [21]. We hope that various antimicrobial breakpoints of $B$. cereus would be set based on the abundant experience with the treatment of $B$. cereus infection. Second, $B$. cereus and $B$. thuringiensis were not distinguished in this study, because the automated identification system was used. Although $B$. thuringiensis is basically a harmless pathogen to humans, the extremely rare case with $B$. thuringiensis infection might have been missed. Third, the study was performed retrospectively based on clinical records, and the number of cases was small. To determine the most effective therapy for $B$. cereus BSI, more large-scale and prospective studies should be performed. 


\section{Conclusions}

The definite BSI of B. cereus accounted for $14.3 \%$ of $B$. cereus-detected cases, and they were mostly caused by venous catheter-related infections. Early clinical resolution was related to appropriate empirical antibiotic therapy. Vancomycin is optimal for empirical therapy of $B$. cereus BSI, but further clinical data are needed.

\section{Authors' contributions}

$\mathrm{Ml}$ and $\mathrm{SO}$ conceived and designed the study, analysed the data, and drafted the manuscript. YY and MO performed microbiological works. MK and KT helped design the study and draft the manuscript. All authors read and approved the final manuscript.

\section{Author details}

${ }^{1}$ Department of Infection Control and Prevention, Faculty of Medicine, The University of Tokyo, 7-3-1 Hongo, Bunkyo, Tokyo 113-8655, Japan. ${ }^{2}$ Department of Medical Technology, School of Health Sciences, Tokyo University of Technology, 5-23-22 Nishikamata, Ota, Tokyo 144-8535, Japan.

\section{Acknowledgements}

The authors wish to thank Dr. Takatoshi Kitazawa and Dr. Yasuo Ota for the discussion on study design.

\section{Compliance with ethical guidelines}

\section{Ethical standard}

This study was approved by the research ethics committee at the University of Tokyo Hospital.

\section{Informed consent}

The requirement of obtaining written informed consent from each patient was waived by the research ethics committee, because this study is retrospective.

\section{Competing interests}

The authors declare that they have no competing interests.

Received: 29 April 2015 Accepted: 31 August 2015

Published online: 15 September 2015

\section{References}

1. Kolst $\varnothing A B$, Tourasse NJ, Økstad OA. What sets Bacillus anthracis apart from other Bacillus species? Annu Rev Microbiol. 2009;63:451-76.

2. Ghelardi E, Celandroni F, Salvetti S, Fiscarelli E, Senesi S. Bacillus thuringiensis pulmonary infection: critical role for bacterial membrane-damaging toxins and host neutrophils. Microbes Infect. 2007;9(5):591-8.

3. Bottone EJ. Bacillus cereus, a volatile human pathogen. Clin Microbiol Rev. 2010;23(2):382-98.

4. Avashia SB, Riggins WS, Lindley C, Hoffmaster A, Drumgoole R, Nekomoto $T$, et al. Fatal pneumonia among metalworkers due to inhalation exposure to Bacillus cereus containing Bacillus anthracis toxin genes. Clin Infect Dis. 2007:44(3):414-6.

5. Rishi E, Rishi P, Sengupta S, Jambulingam M, Madhavan HN, Gopal $L$, et al. Acute postoperative Bacillus cereus endophthalmitis mimicking toxic anterior segment syndrome. Ophthalmology. 2013;120(1):181-5.

6. Gaur A, Patrick C, McCullers J, Flynn P, Pearson T, Razzouk B, et al. Bacillus cereus bacteremia and meningitis in immunocompromised children. Clin Infect Dis. 2001:32(10):1456-62.

7. Hansford JR, Phillips M, Cole C, Francis J, Blyth CC, Gottardo NG. Bacillus cereus bacteremia and multiple brain abscesses during acute lymphoblastic leukemia induction therapy. J Pediatr Hematol Oncol. 2014;36(3):e197-201.

8. Meredith FT, Fowler VG, Gautier M, Corey GR, Reller LB. Bacillus cereus necrotizing cellulitis mimicking clostridial myonecrosis: case report and review of the literature. Scand J Infect Dis. 1997;29(5):528-9.
9. Lee YL, Shih SD, Weng YJ, Chen C, Liu CE. Fatal spontaneous bacterial peritonitis and necrotizing fasciitis with bacteraemia caused by Bacillus cereus in a patient with cirrhosis. J Med Microbiol. 2010;59(Pt 2):242-4.

10. Dellinger RP, Levy MM, Rhodes A, Annane D, Gerlach H, Opal SM, et al. Surviving sepsis campaign: international guidelines for management of severe sepsis and septic shock: 2012. Crit Care Med. 2013;41(2):580-637.

11. Horii T, Notake S, Tamai K, Yanagisawa H. Bacillus cereus from blood cultures: virulence genes, antimicrobial susceptibility and risk factors for blood stream infection. FEMS Immunol Med Microbiol. 2011;63(2):202-9.

12. Ko JH, Kang Cl, Lee WJ, Huh K, Yoo JR, Kim K, et al. Clinical features and risk factors for development of Bacillus bacteremia among adult patients with cancer: a case-control study. Support Care Cancer. 2015:23(2):377-84.

13. Dohmae S, Okubo T, Higuchi W, Takano T, Isobe H, Baranovich T, et al. Bacillus cereus nosocomial infection from reused towels in Japan. J Hosp Infect. 2008;69(4):361-7.

14. Sasahara T, Hayashi S, Morisawa Y, Sakihama T, Yoshimura A, Hirai Y. Bacillus cereus bacteremia outbreak due to contaminated hospital linens. Eur J Clin Microbiol Infect Dis. 2011;30(2):219-26.

15. Inoue $D$, Nagai $Y$, Mori $M$, Nagano $S$, Takiuchi $Y$, Arima $H$, et al. Fulminant sepsis caused by Bacillus cereus in patients with hematologic malignancies: analysis of its prognosis and risk factors. Leuk Lymphoma. 2010;51(5):860-9.

16. Kassar R, Hachem R, Jiang Y, Chaftari A, Raad I. Management of Bacillus bacteremia: the need for catheter removal. Medicine (Baltimore). 2009;88(5):279-83.

17. Uchino Y, Iriyama N, Matsumoto K, Hirabayashi Y, Miura K, Kurita D, et al. A case series of Bacillus cereus septicemia in patients with hematological disease. Intern Med. 2012:51(19):2733-8.

18. Kato K, Matsumura Y, Yamamoto M, Nagao M, Ito Y, Takakura S, et al. Seasonal trend and clinical presentation of Bacillus cereus bloodstream infection: association with summer and indwelling catheter. Eur J Clin Microbiol Infect Dis. 2014:33(8):1371-9.

19. Clinical and Laboratory Standards Institute (CLSI). Methods for antimicrobial dilution and disk susceptibility testing of infrequently isolated or fastidious bacteria; approved guideline, 2nd edn. M45A2E. Wayne: CLSI; 2010.

20. Clinical and Laboratory Standards Institute (CLSI). Performance standards for antimicrobial susceptibility testing; twenty-second informational supplement M100-S22. Wayne: CLSI; 2012.

21. Clinical and Laboratory Standards Institute (CLSI). Performance standards for antimicrobial susceptibility testing; twenty-fourth informational supplement M100-S24. Wayne: CLSI; 2014.

22. Luna VA, King DS, Gulledge J, Cannons AC, Amuso PT, Cattani J. Susceptibility of Bacillus anthracis, Bacillus cereus, Bacillus mycoides, Bacillus pseudomycoides and Bacillus thuringiensis to 24 antimicrobials using Sensititre automated microbroth dilution and Etest agar gradient diffusion methods. J Antimicrob Chemother. 2007;60(3):555-67.

23. Mermel LA, Allon M, Bouza E, Craven DE, Flynn P, O'Grady NP, et al. Clinical practice guidelines for the diagnosis and management of intravascular catheter-related infection: 2009 Update by the Infectious Diseases Society of America. Clin Infect Dis. 2009;49(1):1-45.

24. Hsueh PR, Teng $\sqcup$, Yang PC, Pan HL, Ho SW, Luh KT. Nosocomial pseudoepidemic caused by Bacillus cereus traced to contaminated ethyl alcohol from a liquor factory. J Clin Microbiol. 1999;37(7):2280-4.

25. Loeb M, Wilcox L, Thornley D, Gun-Munro J, Richardson H. Bacillus species pseudobacteremia following hospital construction. Can J Infect Control. 1995; 10(2):37-40

26. López-Cortés LE, Del Toro MD, Gálvez-Acebal J, Bereciartua-Bastarrica E, Fariñas MC, Sanz-Franco M et al. Impact of an evidence-based bundle intervention in the quality-of-care management and outcome of Staphylococcus aureus bacteremia. Clin Infect Dis. 2013:57(9):1225-33.

27. Kang Cl, Kim SH, Park WB, Lee KD, Kim HB, Kim EC, et al. Bloodstream infections due to extended-spectrum beta-lactamase-producing Escherichia coli and Klebsiella pneumoniae: risk factors for mortality and treatment outcome, with special emphasis on antimicrobial therapy. Antimicrob Agents Chemother. 2004;48(12):4574-81.

28. Kiyomizu K, Yagi T, Yoshida H, Minami R, Tanimura A, Karasuno T, et al. Fulminant septicemia of Bacillus cereus resistant to carbapenem in a patient with biphenotypic acute leukemia. J Infect Chemother. 2008:14(5):361-7. 
29. Katsuya H, Takata T, Ishikawa T, Sasaki H, Ishitsuka K, Takamatsu Y, et al. A patient with acute myeloid leukemia who developed fatal pneumonia caused by carbapenem-resistant Bacillus cereus. J Infect Chemother. 2009:15(1):39-41.

30. Sabath LD, Abraham EP. Zinc as a cofactor for cephalosporinase from Bacillus cereus 569. Biochem J. 1966;98(1):11C-3C

31. Rasia RM, Vila AJ. Structural determinants of substrate binding to Bacillus cereus metallo-beta-lactamase. J Biol Chem. 2004;279(25):26046-51.
32. Hilliard N, Schelonka R, Waites K. Bacillus cereus bacteremia in a preterm neonate. J Clin Microbiol. 2003;41(7):3441-4.

33. Salvetti S, Faegri K, Ghelardi E, Kolstø AB, Senesi S. Global gene expression profile for swarming Bacillus cereus bacteria. Appl Environ Microbiol. 2011;77(15):5149-56.

34. Citron DM, Appleman MD. In vitro activities of daptomycin, ciprofloxacin, and other antimicrobial agents against the cells and spores of clinical isolates of Bacillus species. J Clin Microbiol. 2006;44(10):3814-8.

\section{Submit your next manuscript to BioMed Central} and take full advantage of:

- Convenient online submission

- Thorough peer review

- No space constraints or color figure charges

- Immediate publication on acceptance

- Inclusion in PubMed, CAS, Scopus and Google Scholar

- Research which is freely available for redistribution

Submit your manuscript at

www.biomedcentral.com/submit

C Biomed Central 